\title{
CIRCULAR COLOURING AND GRAPH HOMOMORPHISM
}

\author{
XUDING ZHU
}

For any pair of integers $p, q$ such that $(p, q)=1$ and $p \geqslant 2 q$, the graph $G_{p}^{q}$ has vertices
$\{0,1, \ldots, p-1\}$ and edges $\{i j: q \leqslant|i-j| \leqslant p-q\}$. These graphs play the same role in the study of circular chromatic number as that played by the complete graphs in the study of chromatic number. The graphs $G_{p}^{q}$ share many properties of the complete graphs. However, there are also striking differences between the graphs $G_{p}^{q}$ and the complete graphs. We shall prove in this paper that for many pairs of integers $p, q$, one may delete most of the edges of $G_{p}^{q}$ so that the resulting graph still has circular chromatic number $p / q$. To be precise, we shall prove that for any number $r>2$, there exists a rational number $p / q$ (where $(p, q)=1$ ) which is less than $r$ but arbitrarily close to $r$, such that $G_{p}^{q}$ contains a subgraph $H$ with $\chi_{c}(H)=\chi_{c}\left(G_{p}^{q}\right)=p / q$ and $|E(H)|=O\left(\sqrt{\left|E\left(G_{p}^{q}\right)\right|}\right)$. This is in sharp contrast to the fact that the complete graphs are edge critical, that is, the deletion of any edge will decrease its chromatic number and its circular chromatic number.

\section{INTRODUCTION}

All graphs considered in this paper are finite and simple. Suppose $G$ and $H$ are graphs. A homomorphism from $G$ to $H$ is a mapping $f$ from $V(G)$ to $V(H)$ such that $f(x) f(y) \in E(H)$ whenever $x y \in E(G)$. Homomorphisms of graphs are studied as a generalisation of graph colourings. Indeed, a vertex colouring of a graph $G$ with $n$ colours is equivalent to a homomorphism from $G$ to $K_{n}$. We write $G \preceq H$ if there exists a homomorphism from $G$ to $H$. Then $\preceq$ defines a partial order on the set of all finite graphs, which we denote by $(\mathcal{F}, \preceq)$. Two graphs $G$ and $H$ are hom-equivalent, written as $G \sim H$, if $G \preceq H$ and $H \preceq G$. Obviously $\sim$ defines an equivalence relation on the set $\mathcal{F}$. We shall denote by $[G]$ the equivalence class of $\mathcal{F} / \sim$ that contains the graph $G$.

The structure of the partial order $(\mathcal{F} / \sim, \preceq)$ has been extensively studied, $[3,6$, $8,10,11$. For example, there are many density results concerning this partial order $[6,8,10,11]$, and it is known that $(\mathcal{F} / \sim, \preceq)$ forms a distributive lattice $[3]$, et cetera. The study of the chromatic number of graphs can also be viewed as an investigation of

Received 10th June, 1998

This research was partially supported by the National Science under grant NSC87-2115-M-110-004. The paper was finished during the author's visit to Universität Bielefeld.

Copyright Clearance Centre, Inc. Serial-fee code: 0004-9729/99 \$A2.00+0.00. 
the structure of this partial order $(\mathcal{F} / \sim, \preceq)$. We shall denote by $\mathcal{Z}_{\mathcal{G}}$ the set of complete graphs, that is, $\mathcal{Z}_{\mathcal{G}}=\left\{K_{1}, K_{2}, \ldots,\right\}$. Then $\mathcal{Z}_{\mathcal{G}}$ forms an infinite increasing chain in $(\mathcal{F} / \sim, \preceq)$, which maybe viewed as a representation of the natural numbers. Any graph $G \in \mathcal{F}$ admits a homomorphism to some member of the set $\mathcal{Z}_{\mathcal{G}}$. The chromatic number $\chi(G)$ is the minimum $n$ such that $G \preceq K_{n}$, that is, the least element of the set $\mathcal{Z}_{\mathcal{G}}$ which is "above" $G$ in the order $\preceq$ (as we use $K_{n}$ to represent the integer $n$ ). In this sense, we may view the set $\mathcal{Z}_{\mathcal{G}}$ as a scale that measures a dimension of graphs.

Just as the set of natural numbers is extended to the set of positive rationals, we can "extend" the set $\mathcal{Z}_{\mathcal{G}}$ into a larger set. For those fractions $p / q$ with $(p, q)=1$ and $p \geqslant 2 q$, we construct the graph $G_{p}^{q}$, which has vertices $\{0,1, \ldots, p-1\}$ and edges $\{i j: q \leqslant$ $|i-j| \leqslant p-q\}$. We shall denote by $\mathcal{Q}_{\mathcal{G}}$ the set $\left\{G_{p}^{q}:(p, q)=1\right.$ and $\left.p \geqslant 2 q\right\} \cup\left\{K_{1}\right\}$. Note that $G_{p}^{1}=K_{p}$, and hence $\mathcal{Q}_{\mathcal{G}}$ is indeed an extension of $\mathcal{Z}_{\mathcal{G}}$. Moreover, the set $\mathcal{Q}_{\mathcal{G}}$ is also linearly ordered. It was shown in $[2,9]$ that if $p^{\prime} / q^{\prime} \geqslant 2$ and $p / q \geqslant 2$, then $p^{\prime} / q^{\prime} \leqslant p / q$ if and only if $G_{p^{\prime}}^{q^{\prime}} \preceq G_{p}^{q}$. Thus the set $\mathcal{Q}_{\mathcal{G}}$ together with the order $\preceq$ may be viewed as a representation of those rationals $r \geqslant 2$ or $r=1$. The circular chromatic number $\chi_{c}(G)$ of a graph is the infimum of the ratios $p / q$ for which $G \preceq G_{p}^{q}$. It was shown in [9] that the infimum in this definition is always attained, and hence the infimum can be replaced by minimum. Therefore $\chi_{c}(G)$ is the least member of $\mathcal{Q}_{\mathcal{G}}$ which is above $G$ in the order $\preceq$ (as we use $G_{p}^{q}$ to represent the rational $p / q$ ).

If the set $\mathcal{Z}_{\mathcal{G}}$ is considered as a scale that measures a dimension of graphs, then the set $\mathcal{Q}_{\mathcal{G}}$ is a refinement of that scale, just as the set of rational numbers provides a finer scale than that of integers that measures the length of an object. In this sense, the chromatic number $\chi(G)$ of a graph $G$ may be regarded as an approximation of its circular chromatic number $\chi_{c}(G)$. The circular chromatic number of a graph was introduced by Vince [9] in 1988 under the name "the star chromatic number". The concept has enjoyed considerable attention $[1,2,4,7,9,12,13,14,15,16]$.

The graphs $G_{p}^{q}$ share many properties of the complete graphs $K_{n}$. We say a graph $G$ is vertex critical if for any vertex $x$ of $G$, we have $\chi_{c}(G-x)<\chi_{c}(G)$. We say a graph $G$ is edge critical if for any edge $e$ of $G$, we have $\chi_{c}(G-e)<\chi_{c}(G)$. It was shown in $[2,9]$ that for any pair of integers $p, q$ with $p \geqslant 2 q$ and $(p, q)=1$, the graph $G_{p}^{q}$ is vertex critical. It follows that the graphs $G_{p}^{q}$, just like the complete graphs, have the least number of vertices among all the graphs $G$ with $\chi_{c}(G)=p / q$. However, there are also striking differences between the graphs $G_{p}^{q}$ and the complete graphs. It was noted in [12] that for some pairs of integers $(p, q)$, the graphs $G_{p}^{q}$ are not edge critical, while the complete graphs are obviously edge critical. We shall explore this observation further in this paper. We shall determine exactly which graphs $G_{p}^{q}$ are edge critical, and we shall estimate the number of edges that can be deleted from a graph $G_{p}^{q}$ so that the resulting graph still has the same circular chromatic number. Surprisingly, we shall prove that for many pairs of integers $(p, q)$, we can delete most of the edges of $G_{p}^{q}$ without decreasing 
its circular chromatic number. To be precise, we shall prove the following theorems:

TheOREM 1.1. Suppose $p \geqslant 2 q$ and $(p, q)=1$. The graph $G_{p}^{q}$ is edge critical if and only if either $q=1$ or $p=2 q+1$.

THEOREM 1.2. For any number $r>2$, there is a sequence of rational numbers $p_{i} / q_{i}$ (where $\left(p_{i}, q_{i}\right)=1$ ) such that the following is true:

- $p_{i} / q_{i}<r$ and $\lim _{i \rightarrow \infty} p_{i} / q_{i}=r$;

- each of the graphs $G_{p_{i}}^{q_{i}}$ contains a subgraph $H_{i}$ with $\chi_{c}\left(H_{i}\right)=\chi_{c}\left(G_{p_{i}}^{q_{i}}\right)=$ $p_{i} / q_{i}$ and $\left|E\left(H_{i}\right)\right|=O\left(\sqrt{\left|E\left(G_{p_{i}}^{q_{i}}\right)\right|}\right)$.

\section{ThE CONSTRUCTION}

We note that if $q=1$ then $G_{p}^{q}=K_{p}$ is obviously edge critical. If $p=2 q+1$, then $G_{p}^{q}=C_{p}$ is an odd cycle, which is also obviously edge critical. This proves one direction of Theorem 1.1. The rest of this paper is devoted to the proof the other direction of Theorem 1.1 and the proof of Theorem 1.2. We shall first present a systematic way of constructing, for any pair of integers $p \geqslant 2 q$ and $(p, q)=1$, a subgraph $M(p, q)$ of $G_{p}^{q}$ which has the same circular chromatic number as $G_{p}^{q}$. Once the construction is finished, the proofs of Theorems 1.1 and 1.2 are just a comparison of the number of edges of $G_{p}^{q}$ and $M(p, q)$. The method presented here is a generalisation of the method used in [5] and [15] to construct planar graphs with circular chromatic number $r$ for any rational $2 \leqslant r \leqslant 4$. In case $q=1$, then the graph $M(p, q)=G_{p}^{q}=K_{p}$. In the following we only need to consider the case that $p / q$ is not an integer.

For the remaining part of this section, we assume that $p / q>2$ is a fixed rational number and that $(p, q)=1$. Let $m=\lfloor p / q\rfloor$. Thus $p / q$ is strictly between $m$ and $m+1$. To construct the graph $M(p, q)$, we need to construct two auxiliary sequences of numbers: the Farey sequence and the alpha sequence, which are determined by the number $p / q$.

Let $p^{\prime}, q^{\prime}$ be the unique positive integers such that $p^{\prime}<p, q^{\prime}<q$ and $p q^{\prime}-q p^{\prime}=1$. It is straightforward to verify that $p^{\prime} / q^{\prime}<p / q$ and that $p^{\prime} / q^{\prime}$ is the largest fraction with the property that $p^{\prime} / q^{\prime}<p / q$ and $p^{\prime} \leqslant p$. Similarly, we let $p^{\prime \prime}, q^{\prime \prime}$ be positive integers such that $p^{\prime \prime}<p^{\prime}, q^{\prime \prime}<q^{\prime}$ and $p^{\prime} q^{\prime \prime}-p^{\prime \prime} q^{\prime}=1$. Then $p^{\prime \prime} / q^{\prime \prime}$ is the largest fraction with the property that $p^{\prime \prime} / q^{\prime \prime}<p^{\prime} / q^{\prime}$ and $p^{\prime \prime} \leqslant p^{\prime}$. Repeating this process of finding smaller and smaller fractions, we shall stop at the fraction $m / 1$ in a finite number of steps. Thus we obtain a unique sequence of fractions

$$
\frac{m}{1}=\frac{p_{0}}{q_{0}}<\frac{p_{1}}{q_{1}}<\frac{p_{2}}{q_{2}}<\cdots<\frac{p_{n}}{q_{n}}=\frac{p}{q} .
$$

We call the sequence $\left(p_{i} / q_{i}: i=0,1, \ldots, n\right)$ the Farey sequence of $p / q$.

For convenience, we let $p_{-1}=-1$ and $q_{-1}=0$. As $p_{i} q_{i-1}-p_{i-1} q_{i}=1$ and $p_{i-1} q_{i-2}-$ $p_{i-2} q_{i-1}=1$, it follows that $p_{i-1}\left(q_{i}+q_{i-2}\right)=q_{i-1}\left(p_{i}+p_{i-2}\right)$ for $i \geqslant 1$. As $p_{i-1}, q_{i-1}$ are 
co-prime,

$$
\alpha_{i}=\frac{p_{i}+p_{i-2}}{p_{i-1}}=\frac{q_{i}+q_{i-2}}{q_{i-1}}
$$

is an integer (for $i \geqslant 1$ ), which is greater than 1 , and hence is at least 2 . We call $\left(\alpha_{1}, \alpha_{2}, \ldots, \alpha_{n}\right)$ the alpha sequence of $p / q$, which is obviously uniquely determined by $p / q$. The process of deducing the alpha sequence from the rational $p / q$ can also be reversed. In other words, given the integer $m$, each sequence $\left(\alpha_{1}, \alpha_{2}, \ldots, \alpha_{n}\right)$ with $\alpha_{i} \geqslant 2$ determines a rational $p / q$ between $m$ and $m+1$. Indeed, given the alpha sequence $\left(\alpha_{1}, \alpha_{2}, \ldots, \alpha_{n}\right)$, the fractions $p_{i} / q_{i}$ can be easily determined by solving the difference equations

$$
p_{i}=\alpha_{i} p_{i-1}-p_{i-2}, \quad q_{i}=\alpha_{i} q_{i-1}-q_{i-2}
$$

with the initial condition that $\left(p_{-1}, q_{-1}\right)=(-1,0)$ and $\left(p_{0}, q_{0}\right)=(m, 1)$.

Having determined the alpha sequence

$$
\left(\alpha_{1}, \alpha_{2}, \ldots, \alpha_{n}\right)
$$

and the Farey sequence

$$
\frac{m}{1}=\frac{p_{0}}{q_{0}}<\frac{p_{1}}{q_{1}}<\frac{p_{2}}{q_{2}}<\cdots<\frac{p_{n}}{q_{n}}=\frac{p}{q}
$$

of $p / q$, we can start the construction of the graph $M(p, q)$.

We shall construct a sequence of graphs $G_{i}$ such that $\chi_{c}\left(G_{i}\right)=p_{i} / q_{i}$. Then $M(p, q)=$ $G_{n}$. Before constructing the graphs $G_{i}$, we shall recursively construct ordered graphs $F_{i}, H_{i}$, that is, the vertices of $F_{i}$ and $H_{i}$ are linearly ordered. Let $f_{i}=\left|F_{i}\right|$ and $h_{i}=\left|H_{i}\right|$, then the vertices of $F_{i}$ will be denoted by $\left(x_{i, 1}, x_{i, 2}, \ldots, x_{i, f_{i}}\right)$ in that order, and the vertices of $H_{i}$ will be denoted by $\left(y_{i, 1}, y_{i, 2}, \ldots, y_{i, h_{i}}\right)$ in that order. For an edge $e=(x, y)$ of an ordered graph, we define the order length of $e$, denoted by $\ell(e)$, to be the positive difference between the positions of $x$ and $y$.

DEF IN ITION 2.1. Suppose $X$ and $Y$ are disjoint ordered graphs whose vertex orderings are $\left(x_{1}, x_{2}, \ldots, x_{s}\right)$ and $\left(y_{1}, y_{2}, \ldots, y_{t}\right)$, respectively. When we say hook $X$ to $Y$, it means to add the following edges between $X$ and $Y$ :

$$
x_{1} y_{t}, x_{1} y_{t-1}, \ldots, x_{1} y_{t-m+2}, x_{s} y_{1}, x_{s} y_{2}, x_{s} y_{3}, \ldots, x_{s} y_{m-1}
$$

The result of hooking a sequence $X_{1}, X_{2}, \ldots, X_{\beta}$ of ordered graphs is regarded as another ordered graph, the order of the vertices being: those of $X_{1}$ in order, followed by those of $X_{2}$ in order, and so on.

For an integer $t$, we let $Q_{t}$ be the $(m-1)$ th power of the path of length $t-1$, that is, $Q_{t}$ has vertex set $\left\{v_{1}, v_{2}, \ldots, v_{t}\right\}$ in which two vertices $v_{i}$ and $v_{j}$ are adjacent if $|i-j| \leqslant m-1$. The graph $Q_{t}$ is considered as an ordered graph in the following, where the order of the vertices is $\left(v_{1}, v_{2}, \ldots, v_{t}\right)$. 
First of all, we let $F_{1}$ be a singleton, let $H_{1}=Q_{m \alpha_{1}}$, and let $F_{2}=Q_{m\left(\alpha_{1}-1\right)}$.

For $i \geqslant 1$, to construct the graph $H_{i+1}$, we take $\alpha_{i+1}$ copies of $F_{i}$, denoted by $F_{i}^{1}, F_{i}^{2}, \ldots, F_{i}^{\alpha_{i+1}}$, and $\alpha_{i+1}-1$ copies of $H_{i}$, denoted by $H_{i}^{1}, H_{i}^{2}, \ldots, H_{i}^{\alpha_{i+1}-1}$. If $i$ is odd, then for $j=1,2, \ldots, \alpha_{i+1}-1$, we hook $F_{i}^{j}$ and $F_{i}^{j+1}$ to $H_{i}^{j}$. If $i$ is even, then for $j=1,2, \ldots, \alpha_{i+1}-1$, we hook $H_{i}^{j}$ to $F_{i}^{j}$ and $F_{i}^{j+1}$. The resulting graph is $H_{i+1}$. The graph $F_{i+2}$ is constructed in the same way as the graph $H_{i+1}$, but with one less copy of $F_{i}$ and $H_{i}$, that is, $F_{i+2}$ is constructed from $\alpha_{i+1}-1$ copies of $F_{i}$ and $\alpha_{i+1}-2$ copies of $H_{i}$, by appropriately hooking them together.

Finally when $i$ is even, we let $G_{i}$ be the graph obtained by hooking $H_{i}$ to $F_{i}$; when $i$ is odd, we let $G_{i}$ be the graph obtained by hooking $F_{i}$ to $H_{i}$.

This finishes the construction of the graphs $G_{i}$. The graph $M(p, q)$ is equal to $G_{n}$. We note that when $m=2$, the graphs constructed here are the same as constructed by Moser in [5].

\section{THE CIRCULAR CHROMATIC NUMBER}

In this section, we shall prove that $M(p, q)=G_{n}$ is a subgraph of $G_{p}^{q}$ and that $\chi_{c}(M(p, q))=p / q$.

First we count the number of vertices of $G_{i}$.

LEMMA 3.1. The graph $G_{i}$ has $p_{i}$ vertices.

Proof: From the construction of $G_{i}$, we know that $G_{i}$ has $g_{i}=f_{i}+h_{i}$ vertices. From the construction of $F_{i}, H_{i}$, we know that

$$
f_{1}=1, \quad f_{2}=m \alpha_{1}-m, \quad h_{1}=m \alpha_{1},
$$

and for $i \geqslant 2$,

$$
h_{i}=\alpha_{i} f_{i-1}+\left(\alpha_{i}-1\right) h_{i-1},
$$

for $i \geqslant 3$,

$$
f_{i}=\left(\alpha_{i-1}-1\right) f_{i-2}+\left(\alpha_{i-1}-2\right) h_{i-2} .
$$

Simple algebraic calculation shows that

$$
h_{i}=\alpha_{i} g_{i-1}-h_{i-1}, f_{i}=\left(\alpha_{i-1}-1\right) g_{i-2}-h_{i-2}=h_{i-1}-g_{i-2}
$$

Hence

$$
g_{i}=\alpha_{i} g_{i-1}-g_{i-2}
$$

Since $g_{1}=p_{1}, g_{2}=p_{2}$, and $g_{i}, p_{i}$ satisfy the same difference equation, we conclude that $\left|G_{i}\right|=g_{i}=p_{i}$.

Next we shall show that $M(p, q)$ is a subgraph of $G_{p}^{q}$. For this purpose, we shall prove by induction that for each $i \leqslant n$, the graph $G_{i}$ is a subgraph of $G_{p_{i}}^{q_{i}}$. 
Before proving this, we need some preliminary results about the relation between the Farey sequence and the alpha sequence. We observed before that the Farey sequence is uniquely determined by the alpha sequence. The numbers $p_{i}$ and $q_{i}$ are obtained by solving the following difference equations:

$$
p_{i}=\alpha_{i} p_{i-1}-p_{i-2}, \quad q_{i}=\alpha_{i} q_{i-1}-q_{i-2}
$$

with the initial condition that $\left(p_{-1}, q_{-1}\right)=(-1,0)$ and $\left(p_{0}, q_{0}\right)=(m, 1)$.

By repeatedly applying the equation $(*)$, we may express $p_{i}$ (respectively $q_{i}$ ) in terms of $p_{j}$ and $p_{j-1}$ (respectively $q_{j}$ and $q_{j-1}$ ) for any $0 \leqslant j \leqslant i-2$. Lemma 3.2 below gives the explicit expressions.

For $1 \leqslant r \leqslant s \leqslant n$, we let

$$
\Lambda_{r, s}=\operatorname{det}\left(\begin{array}{cccccc}
\alpha_{r} & 1 & 0 & \cdots & 0 & 0 \\
1 & \alpha_{r+1} & 1 & \cdots & 0 & 0 \\
0 & 1 & \alpha_{r+2} & \cdots & 0 & 0 \\
\vdots & \vdots & \vdots & \vdots & \vdots & \vdots \\
0 & 0 & 0 & \cdots & \alpha_{s-1} & 1 \\
0 & 0 & 0 & \cdots & 1 & \alpha_{s}
\end{array}\right)
$$

LEMMA 3.2. For $0 \leqslant j \leqslant i-2$, we have

$$
p_{i}=p_{j} \Lambda_{j+1, i}-p_{j-1} \Lambda_{j+2, i,} \quad q_{i}=q_{j} \Lambda_{j+1, i}-q_{j-1} \Lambda_{j+2, i}
$$

PROOF: It suffices to prove the first equality. We shall prove it by induction on $i$. When $i=j+2$, by applying $(*)$ twice, we obtain $(* *)$. Suppose $i \geqslant j+3$, and that $(* *)$ is true for any $i^{\prime}<i$. Then by cofactor expansion,

$$
\begin{aligned}
p_{j} \Lambda_{j+1, i}-p_{j-1} \Lambda_{j+2, i} & =\alpha_{i}\left(p_{j} \Lambda_{j+1, i-1}-p_{j-1} \Lambda_{j+2, i-1}\right)-\left(p_{j} \Lambda_{j+1, i-2}-p_{j-1} \Lambda_{j+2, i-2}\right) \\
& =\alpha_{i} p_{i-1}-p_{i-2}=p_{i} .
\end{aligned}
$$

The second equality uses the induction hypothesis.

By letting $j=0$ in $(* *)$, and by using the initial condition, we have

$$
p_{i}=m \Lambda_{1, i}+\Lambda_{2, i}, \quad q_{i}=\Lambda_{1, i} .
$$

LEMMA 3.3. For $0 \leqslant j \leqslant i-2, p_{j} q_{i}=p_{i} q_{j}-\Lambda_{j+2, i}$.

ProOF: By applying Lemma 3.2, we have

$$
\begin{aligned}
p_{i} q_{j}-p_{j} q_{i} & =\left(p_{j} \Lambda_{j+1, i}-p_{j-1} \Lambda_{j+2, i}\right) q_{j}-p_{j}\left(q_{j} \Lambda_{j+1, i}-q_{j-1} \Lambda_{j+2, i}\right) \\
& =\Lambda_{j+2, i}\left(p_{j} q_{j-1}-p_{j-1} q_{j}\right) \\
& =\Lambda_{j+2, i} .
\end{aligned}
$$


LEMMA 3.4. For any $2<t \leqslant i, \Lambda_{t, i}<\Lambda_{t-1, i}$.

We omit the proof, which is an easy induction, by noting that $\alpha_{j} \geqslant 2$.

Let the vertices of $G_{p_{i}}^{q_{i}}$ be $\left\{0,1, \ldots, p_{i}-1\right\}$. We shall define a 1-1 mapping $c$ from $V\left(G_{i}\right)$ to $V\left(G_{p_{i}}^{q_{i}}\right)$ as follows:

We know that the vertex set of $G_{i}$ is the union of the vertex set of $F_{i}$ and the vertex set of $H_{i}$, while the vertices of $F_{i}$ are $\left(x_{i, 1}, x_{i, 2}, \ldots, x_{i, f_{i}}\right)$ and the vertices of $H_{i}$ are $\left(y_{i, 1}, y_{i, 2}, \ldots, y_{i, h_{i}}\right)$. We shall rename the vertices of $G_{i}$ by letting $v_{j}=x_{i, j}$ for $j=$ $1,2, \ldots, f_{i}$, and let $v_{j}=y_{i, j-f_{i}}$ for $j=f_{i}+1, f_{i}+2, \ldots, f_{i}+h_{i}\left(=p_{i}\right)$.

Let $c\left(v_{j}\right)=j q_{i}\left(\bmod p_{i}\right)$. As $p_{i}$ and $q_{i}$ are coprime, we know that $c$ is a $1-1$ mapping. Now we shall show that for any edge $\left(v_{j}, v_{j^{\prime}}\right)$ of $G_{i},\left(c\left(v_{j}\right), c\left(v_{j^{\prime}}\right)\right)$ is an edge of $G_{p_{i}}^{q_{i}}$, that is, $q_{i} \leqslant\left|c\left(v_{j}\right)-c\left(v_{j^{\prime}}\right)\right| \leqslant p_{i}-q_{i}$.

First we determine the order length of all edges of $G_{i}$. Recall that the order length $\ell(e)$ of an edge $e=(x, y)$ is the positive difference of the positions of $x$ and $y$ in the above ordering of the vertices of $G_{i}$, that is, if $e=\left(v_{i}, v_{j}\right)$ then $\ell(e)=|i-j|$.

LEMMA 3.5. Let $L=\left\{p_{j}-s: 0 \leqslant j \leqslant i, 1 \leqslant s \leqslant m-1\right\}$. Then for any edge $e$ of $G_{i}$, we have $\ell(e) \in L$.

ProOF: This is easily proved by induction. Each edge of $H_{1}$ or $F_{2}$ has order length $m-s=p_{0}-s$ for some $1 \leqslant s \leqslant m-1$. Suppose the order length of edges in $H_{j}$ and $F_{j}$ are elements of $L$. When copies of $F_{j}$ and $H_{j}$ are hooked together to form $H_{j+1}$ or $F_{j+2}$, then the order length of the edges in the copies of $F_{j}$ and $H_{j}$ are unchanged. For those edges $e$ of the hooks, it is easy to verify that either $\ell(e) \in\{1,2, \ldots, m-1\}$ or $\ell(e) \in\left\{p_{j}-1, p_{j}-2, \ldots, p_{j}-m+1\right\}$. This completes the proof of Lemma 3.5.

LEMMA 3.6. For each $i \leqslant n$, the graph $G_{i}$ is a subgraph of $G_{p_{i}}^{q_{i}}$.

PROOF: It suffices to show that the mapping $c$ defined above is edge preserving, that is, for any edge $(x, y)$ of $G_{i}, q_{i} \leqslant|c(x)-c(y)| \leqslant p_{i}-q_{i}$.

Suppose $e=(x, y)$ is an edge of $G_{i}$ of order length $\ell(e)$. Then by the definition, we have $|c(x)-c(y)|=\ell(e) q_{i} \quad\left(\bmod p_{i}\right)$. By Lemma $3.5, \ell(e) \in L$.

By Lemma 3.3 , for any $0 \leqslant j \leqslant i-2$, we have

$$
p_{j} q_{i}=p_{i} q_{j}-\Lambda_{j+2, i}
$$

By Lemma 3.4, $(* * *)$ and the definition of $\Lambda_{r, s}$, and by noting that $p_{0}=m, q_{0}=1$, we have

$$
2 \leqslant \alpha_{i}=\Lambda_{i, i} \leqslant \Lambda_{j+2, i} \leqslant \Lambda_{2, i}=p_{i}-m q_{i} .
$$

This implies that for any $0 \leqslant j \leqslant i-2$ and $1 \leqslant s \leqslant m-1$,

$$
q_{i}=p_{i}-(m-1) q_{i}-\Lambda_{2, i} \leqslant p_{i}-s q_{i}-\Lambda_{j+2, i} \leqslant p_{i}-s q_{i}-2<p_{i}-q_{i} .
$$


Now if $\ell(e)=p_{j}-s$ where $0 \leqslant j \leqslant i-2$ and $1 \leqslant s \leqslant m-1$, then by Lemma 3.3, we have

$$
|c(x)-c(y)| \equiv\left(p_{j}-s\right) q_{i} \equiv p_{i}-s q_{i}-\Lambda_{j+2, i} \quad\left(\bmod p_{i}\right),
$$

and so $q_{i} \leqslant|c(x)-c(y)| \leqslant p_{i}-q_{i}$, as required.

If $\ell(e)=p_{i-1}-s$ for some $1 \leqslant s \leqslant m-1$, then since $p_{i} q_{i-1}-p_{i-1} q_{i}=1$ by the definition of the Farey sequence, we also have

$$
q_{i} \leqslant \ell(e) q_{i} \quad\left(\bmod p_{i}\right) \leqslant p_{i}-q_{i}
$$

If $\ell(e)=p_{i}-s$ for some $1 \leqslant s \leqslant m-1$, then trivially we have

$$
q_{i} \leqslant \ell(e) q_{i} \quad\left(\bmod p_{i}\right) \leqslant p_{i}-q_{i}
$$

As $M(p, q)=G_{n}$, it follows that $M(p, q)$ is a subgraph of $G_{p}^{q}$. Next we shall prove that $\chi_{c}(M(p, q))=p / q$. This is achieved by showing recursively that for each $i, \chi_{c}\left(G_{i}\right)=$ $p_{i} / q_{i}$.

As $G_{i}$ is a subgraph of $G_{p_{i}}^{q_{i}}$, it follows that $\chi_{c}\left(G_{i}\right) \leqslant p_{i} / q_{i}$. Thus it suffices to show that $\chi_{c}\left(G_{i}\right) \geqslant p_{i} / q_{i}$. First we need a few lemmas.

Lemma 3.7 below was proved in [4] and also implicitly used in $[9,12]$. Suppose $k \geqslant 2 d$ are integers and $(k, d)=1$. We call a homomorphism of $G$ to the graph $G_{k}^{d}$ a $(k, d)$-colouring of $G$.

Given a $(k, d)$-colouring $c$ of a graph $G$, we define a directed graph $D_{c}(G)$ on the vertex set of $G$ by putting a directed edge from $x$ to $y$ if and only if $(x, y)$ is an edge of $G$ and that $c(x)-c(y)=d(\bmod k)$.

LEMMA 3.7. For any graph $G, \chi_{c}(G)=k / d$ if and only if $G$ is $(k, d)$-colourable, and for any $(k, d)$-colouring $c$ of $G$, the directed graph $D_{c}(G)$ contains a directed cycle. $\square$

A simple calculation shows that the length of the directed cycle in $D_{c}(G)$ is a multiple of $k$, and hence is at least $k$.

Corollary 3.1. For any graph $G$, if $\chi_{c}(G)=k / d$ where $(k, d)=1$, then $G$ has a cycle of length at least $k$. In particular $k \leqslant|V(G)|$.

Suppose $\chi_{c}\left(G_{i}\right)=p_{i} / q_{i}$, and that $\Delta$ is an $\left(p_{i}, q_{i}\right)$-colouring of $G_{i}$. It follows from Lemma 3.7 that there is a directed cycle of $D_{\Delta}\left(G_{i}\right)$ of length at least $p_{i}$. Since $\left|G_{i}\right|=p_{i}$, we conclude that there is a Hamiltonian cycle, say $Q=\left(c_{1}, c_{2}, \ldots, c_{p_{i}}, c_{1}\right)$, of $G_{i}$ such that $\Delta\left(c_{j}\right)-\Delta\left(c_{j-1}\right)=q_{i}\left(\bmod p_{i}\right)$.

We say a Hamiltonian cycle $Q=\left(c_{1}, c_{2}, \ldots, c_{t}, c_{1}\right)$ of the graph $G$ is a good Hamiltonian cycle (with respect to $p / q$ ) if for any edge $\left(c_{k}, c_{s}\right)$ of $G$ we have $k-s \neq m, m+1$. Similarly a Hamiltonian path $P=\left(c_{1}, c_{2}, \ldots, c_{t}\right)$ of a graph $G$ is a good Hamiltonian path if for any edge $\left(c_{k}, c_{\ell}\right)$ of $G$, we have $k-\ell \neq m, m+1$. 
Now we shall show that if $\chi_{c}\left(G_{i}\right)=p_{i} / q_{i}$, then the Hamiltonian cycle induced by any $\left(p_{i}, q_{i}\right)$-colouring of $G_{i}$ is a good Hamiltonian cycle.

Lemma 3.8. Suppose $\chi_{c}\left(G_{i}\right)=p_{i} / q_{i}$ and that $\Delta$ is an $\left(p_{i}, q_{i}\right)$-colouring of $G_{i}$. Let $Q=\left(c_{1}, c_{2}, \ldots, c_{p_{i}}, c_{1}\right)$ be a Hamiltonian cycle of $G_{i}$ such that $\Delta\left(c_{j}\right)-\Delta\left(c_{j-1}\right)=q_{i}$ $\left(\bmod p_{i}\right)$. Then $Q$ is a good Hamiltonian cycle of $G_{i}$.

Proof: Assume to the contrary that there is an edge $\left(c_{k}, c_{s}\right)$ of $G_{i}$ such that $|k-s|=$ $m$ or $m+1$. Then $\left|\Delta\left(c_{k}\right)-\Delta\left(c_{s}\right)\right|=m q_{i} \quad\left(\bmod p_{i}\right)$ or $(m+1) q_{i} \quad\left(\bmod p_{i}\right)$. However, $p_{i}-q_{i}<m q_{i}<p_{i}$ and $(m+1) q_{i}\left(\bmod p_{i}\right)<q_{i}$, because $m<p_{i} / q_{i}<m+1$. This is contrary to the assumption that $\Delta$ is a $\left(p_{i}, q_{i}\right)$-colouring of $G_{i}$.

LEMMA 3.9. If $i$ is odd (respectively even), then for any good Hamiltonian path $P$ of $H_{i}$ (respectively $F_{i}$ ), the first $(m-1)$ vertices of $P$ (as a set) are the first $(m-1)$ vertices of $H_{i}$ (respectively $F_{i}$ ), and the last $(m-1)$ vertices of $P$ (as a set) are the last $(m-1)$ vertices of $H_{i}$ (respectively $F_{i}$ ). Here we may reverse the order of all the vertices of $P$, if needed.

Proof: First we consider the graphs $H_{1}$ and $F_{2}$. Each of them is of the form $Q_{t}$ for some positive integer $t$. We shall simply prove that for any positive integer $t$, the graph $Q_{t}$ has a unique good Hamiltonian path, up to an isomorphism. When $m=2$, then $Q_{t}$ is simply a path, and there is nothing to be proved. When $t \leqslant m$, then $Q_{t}$ is a complete graph, and there is also nothing to be proved. Assume now that $t \geqslant m+1$ and $m \geqslant 3$. Suppose the vertices of $Q_{t}$ are $1,2, \ldots, t$, where $(x, y)$ is an edge if and only if $|x-y| \leqslant m-1$. Let $P=\left(x_{1}, x_{2}, \ldots, x_{t}\right)$ be a good Hamiltonian path of $Q_{t}$. Then for any edge $\left(x_{i}, x_{j}\right)$ of $Q_{t}$, we have $|i-j| \neq m, m+1$. This, in particular, implies that for any $i \leqslant t-m$, the pair $\left(x_{i}, x_{i+m}\right)$ is not an edge of $Q_{t}$. In other words, for any $i \leqslant t-m$, $\left|x_{i}-x_{m+i}\right| \geqslant m$.

We shall assume that $x_{1}<x_{m+1}$. The case that $x_{1}>x_{m+1}$ can be treated similarly. (In that case, we need to reverse the order of all the vertices of $P$.) Since $\left|x_{1}-x_{m+1}\right| \geqslant m$, we have $x_{1} \leqslant x_{m+1}-m$. Because $x_{2} \leqslant x_{1}+m-1$ and $x_{m+2} \geqslant x_{m+1}-m+1$ ( as $\left(x_{1}, x_{2}\right)$ and $\left(x_{m+1}, x_{m+2}\right)$ are edges of $\left.Q_{t}\right)$, we conclude that $x_{2} \leqslant x_{m+2}+m-2$. Since $\left|x_{2}-x_{m+2}\right| \geqslant m$, we conclude that $x_{2} \leqslant x_{m+2}-m$. Repeating this argument, we can prove that $x_{i} \leqslant x_{i+m}-m$ for all $i \leqslant t-m$. This implies that $\left\{x_{1}, x_{2}, \ldots, x_{m}\right\}=\{1,2, \ldots, m\}$, for otherwise there would exist an $x \leqslant m$ and an $i \geqslant 1$ such that $x_{i+m}=x$ and hence $1 \leqslant x_{i} \leqslant x_{i+m}-m=x-m \leqslant 0$, an obvious contradiction.

Suppose $x_{i}=m+1$. Then $i \geqslant m+1$, by the previous paragraph. Since $x_{i-m} \leqslant$ $x_{i}-m=1$, we conclude that $x_{i-m}=1$. If $i-m>1$, then $2 \leqslant x_{i-m-1} \leqslant m$ and hence $\left|x_{i}-x_{i \sim m-1}\right| \leqslant m-1$ and $x_{i-m-1} x_{i}$ is an edge of $Q_{t}$, contrary to the assumption that $P$ is a good Hamiltonian path. Therefore we have $x_{1}=1$ and $x_{m+1}=m+1$.

Now we shall prove by induction that for all $1 \leqslant i \leqslant \min \{m, t-m\}$, we have $x_{i}=i$ and $x_{i+m}=i+m$. When $i=1$, this has been proved in the previous paragraph. Assume 
that for any $1 \leqslant j<i$, we have $x_{j}=j$ and $x_{j+m}=j+m$. Let $i+m=x_{k}$. By the previous discussion and the induction hypotheses, we know that $k \geqslant i+m$. Since $x_{k-m} \leqslant x_{k}-m=i$, we conclude that $x_{k-m}=i$, because $x_{k-m} \neq j$ for any $j<i$ by the induction hypotheses. If $k>i+m$, then $i<x_{k-m-1} \leqslant m$ (by using the induction hypotheses). This implies that $\left(x_{k-m-1}, x_{k}\right)$ is an edge of $Q_{t}$, contrary to the assumption that $P$ is a good Hamiltonian path. Therefore we must have $x_{i}=i$ and $x_{i+m}=i+m$, for all $i \leqslant \min \{m, t-m\}$. If $t \geqslant 2 m$, then we have $x_{i}=i$ for all $1 \leqslant i \leqslant t$. If $t=m+j$ for some $1<j<m$, then $x_{i}=i$ except possibly where $j+1 \leqslant i \leqslant m$. But each vertex labelled $j+1, \ldots, m$ is adjacent to every vertex of $Q_{t}$ except itself, so an arbitrary permutation of these vertices gives an automorphism of $Q_{t}$.

This finishes the proof that any good Hamiltonian path of $H_{1}$ (respectively $F_{2}$ ) has the same first $(m-1)$ vertices and the same $(m-1)$ last vertices as $H_{1}$ (respectively $F_{2}$ ).

Assume that the lemma is true for $i$. We shall show that it is true for $i+1$. First we consider the case that $i$ is even. The graph $H_{i+1}$ is obtained by approriately hooking copies of $H_{i}$ to copies of $F_{i}$ (see the construction in Section 2).

Since the first and the last vertex of each copy of $H_{i}$ (in $H_{i+1}$ ) form a 2-vertex cut of $H_{i+1}$, we conclude that any good Hamiltonian path of $H_{i+1}$ is the concatenation of good Hamiltonian paths of the copies of $F_{i}$ and $H_{i}$. Therefore the first $(m-1)$ vertices of any good Hamiltonian path of $H_{i+1}$ are the first $(m-1)$ vertices of a good Hamiltonian path of the first copy of $F_{i}$. By the induction hypothesis, these $(m-1)$ vertices are the first $(m-1)$ vertices of the first copy of $F_{i}$, which by definition are the first $(m-1)$ vertices of $H_{i+1}$. Similarly the last $(m-1)$ vertices of any good Hamiltonian path of $H_{i+1}$ are the last $(m-1)$ vertices of $H_{i+1}$.

The case that $i$ is odd can be treated similarly, and is omitted.

LEMMA 3.10. Suppose $\chi_{c}\left(G_{i}\right)=p_{i} / q_{i}$ for some $i$. Let $\Delta$ be any $\left(p_{i}, q_{i}\right)$-colouring of $G_{i}$. If $i$ is odd, then the colours of the first and last vertices of $F_{i}$ uniquely determine the colours of the first and last $(m-1)$ vertices of $H_{i}$. Conversely, the colours of the first and last $(m-1)$ vertices of $H_{i}$ uniquely determine the colours of the first and last vertices of $F_{i}$. If $i$ is even, then the colours of the first and last vertices of $H_{i}$ uniquely determine the colours of the first and last $(m-1)$ vertices of $F_{i}$. Conversely, the colours of the first and last $(m-1)$ vertices of $F_{i}$ uniquely determine the colours of the first and last vertices of $F_{i}$.

PROOF: We only consider the case that $i$ is odd. Let $\Delta$ be a $\left(p_{i}, q_{i}\right)$-colouring of $G_{i}$. By Lemma 3.8, there is a good Hamiltonian cycle $Q=\left(c_{1}, c_{2}, \ldots, c_{p_{i}}, c_{1}\right)$ of $G_{i}$ such that $\Delta\left(c_{j}\right)-\Delta\left(c_{j-1}\right)=q_{i} \quad\left(\bmod p_{i}\right)$.

The graph $G_{i}$ is obtained by hooking $F_{i}$ to $H_{i}$. The first and the last vertex of $F_{i}$ form a 2-vertex cut of $G_{i}$. Therefore the good Hamiltonian cycle $Q$ is the union of a good Hamiltonian path $P$ of $H_{i}$ and a good Hamiltonian path $P^{\prime}$ of $F_{i}$. By Lemma 3.9, 
the first $(m-1)$ vertices of $P$ are the first $(m-1)$ vertices of $H_{i}$, and the last $(m-1)$ vertices of $P$ are the last $(m-1)$ vertices of $H_{i}$. As the first and the last vertex of $F_{i}$ form a 2-vertex cut of $G_{i}$, the first and last vertex of $P^{\prime}$ must be the first and last vertex of $F_{i}$, respectively. Therefore the colours of the first and last vertex of $F_{i}$ are uniquely determined by the colours of the first and last $(m-1)$ vertices of $H_{i}$, and that the first and last $(m-1)$ vertices of $H_{i}$ are uniquely determined by the colours of the first and last vertices of $F_{i}$.

To prove that $\chi_{c}\left(G_{i}\right) \geqslant p_{i} / q_{i}$ (and hence $\left.\chi_{c}\left(G_{i}\right)=p_{i} / q_{i}\right)$, we need another gadget. If $i \geqslant 2$ is even, let $T_{i}$ be the graph obtained by hooking $F_{i-1}$ to $F_{i}$. If $i \geqslant 2$ is odd, let $T_{i}$ be the graph obtained by hooking $F_{i}$ to $F_{i-1}$.

THEOREM 3.1. For each $i \geqslant 2, \chi_{c}\left(G_{i}\right)=p_{i} / q_{i}$ and $\chi_{c}\left(T_{i}\right)>p_{i-1} / q_{i-1}$. Moreover, $\chi_{c}\left(G_{1}\right)=p_{1} / q_{1}$.

Proof: First we prove that $\chi_{c}\left(G_{1}\right)=p_{1} / q_{1}$. By Lemma 3.6, it suffices to show that $\chi_{c}\left(G_{1}\right) \geqslant p_{1} / q_{1}$. It is easy to verify that $\chi\left(G_{1}\right)=m+1$. Hence $\chi_{c}\left(G_{1}\right)>m$. Suppose $\chi_{c}\left(G_{1}\right)=k / d>m$, then $k \leqslant\left|V\left(G_{1}\right)\right|=p_{1}$ by Corollary 3.1. Therefore $k / d \geqslant p_{1} / q_{1}$, because it follows from the construction of the Farey sequence that any fraction $a / b$ strictly between $m=p_{0} / q_{0}$ and $p_{1} / q_{1}$ must have numerator $a>p_{1}$.

Next we show that $\chi_{c}\left(T_{2}\right)>p_{1} / q_{1}$. Again it is easy to verify that $\chi\left(T_{2}\right)=m+1$. Suppose $\chi_{c}\left(T_{2}\right)=k / d>m$. As $\left|V\left(T_{2}\right)\right|<p_{1}$ (because $\left.\left|V\left(F_{2}\right)\right|<\left|V\left(H_{1}\right)\right|\right)$, we know that $k<p_{1}$. Therefore $k / d>p_{1} / q_{1}$, because by the construction of the Farey sequence, any fraction $a / b$ strictly between $m$ and $p_{1} / q_{1}$ has numerator $a>p_{1}$ (note that $k / d \neq p_{1} / q_{1}$ ).

Now assume that $i \geqslant 2, \chi_{\mathrm{c}}\left(T_{i}\right)>p_{i-1} / q_{i-1}$ and that $\chi_{c}\left(G_{i-1}\right)=p_{i-1} / q_{i-1}$. We shall prove that $\chi_{c}\left(G_{i}\right)=p_{i} / q_{i}$.

Assume to the contrary that $\chi_{c}\left(G_{i}\right)=k / d<p_{i} / q_{i}$ and $(k, d)=1$. Then $k \leqslant p_{i}$ and hence $k / d \leqslant p_{i-1} / q_{i-1}$, because by the construction of the Farey sequence, any fraction $a / b$ strictly between $p_{i-1} / q_{i-1}$ and $p_{i} / q_{i}$ has numerator $a>p_{i}$. Since $\chi_{c}\left(G_{i-1}\right)=p_{i-1} / q_{i-1}$ and that $G_{i-1}$ is a subgraph of $G_{i}$, it follows that $\chi_{c}\left(G_{i}\right)=p_{i-1} / q_{i-1}$.

Let $\Delta$ be a $\left(p_{i-1}, q_{i-1}\right)$-colouring of $G_{i}$. The graph $G_{i}$ is obtained by hooking $H_{i}$ and $F_{i}$ together, and $H_{i}$ is constructed from $\alpha_{i}$ copies of $F_{i-1}$ and $\alpha_{i}-1$ copies of $H_{i-1}$. The union of the first copy of $H_{i-1}$ the first copy of $F_{i-1}$ induces a $G_{i-1}$. The union of the first copy of $H_{i-1}$ and the second copy of $F_{i-1}$ also induces a $G_{i-1}$.

Assume first that $i$ is odd. By using the induction hypotheses that $\chi_{c}\left(G_{i-1}\right)=$ $p_{i-1} / q_{i-1}$, and by applying Lemma 3.10 to each of the two copies of $G_{i-1}$, we conclude that the last $(m-1)$ vertices of the first copy of $F_{i-1}$ are coloured the same way as the last $(m-1)$ vertices of the second copy of $F_{i-1}$. Similarly, the first and last vertices of the first copy of $H_{i-1}$ are coloured the same way as the first and last vertices of the second copy of $H_{i-1}$. Repeating the same argument, we conclude that the last $(m-1)$ vertices of the first copy $F_{i-1}$ are coloured the same way as the last $(m-1)$ vertices of the last copy of $F_{i-1}$ of $H_{i}$. 
This implies that the restriction of $\Delta$ to the union of $F_{i}$ and the first copy of $F_{i-1}$ in $H_{i}$ is indeed a $\left(p_{i-1}, q_{i-1}\right)$-colouring of $T_{i}$, contrary to our assumption that $\chi_{c}\left(T_{i}\right)>p_{i-1} / q_{i-1}$. The case that $i$ is even can be treated similarly.

Finally, assuming that $i \geqslant 2, \chi_{i}\left(G_{i}\right)=p_{i} / q_{i}$ and that $\chi_{c}\left(T_{i}\right)>p_{i-1} / q_{i-1}$, we shall prove that $\chi_{\boldsymbol{c}}\left(T_{i+1}\right)>p_{i} / q_{i}$.

Assume to the contrary that $\chi_{c}\left(T_{i+1}\right)=k / d \leqslant p_{i} / q_{i}$. Since $\left|F_{i+1}\right|<\left|H_{i}\right|$, hence $\left|T_{i+1}\right|<\left|G_{i}\right|=p_{i}$. It follows from Corollary 3.1 that $k<p_{i}$. As $p_{i-1} / q_{i-1}$ is the largest fraction with the property that $p_{i-1}<p_{i}$ and $p_{i-1} / q_{i-1} \leqslant p_{i} / q_{i}$, we conclude that $\chi_{c}\left(T_{i+1}\right) \leqslant p_{i-1} / q_{i-1}$.

We consider two cases:

CASE 1. $\alpha_{i}=2$. In this case $F_{i+1}=F_{i-1}$, and hence $T_{i+1}=T_{i}$. By the induction hypothesis, $\chi_{c}\left(T_{i}\right)>p_{i-1} / q_{i-1}$.

CASE 2. $\alpha_{i}>2$. In this case $F_{i+1}$ consists of $\alpha_{i}-1$ copies of $F_{i-1}$ and $\alpha_{i}-2$ copies of $H_{i-1}$. The union of any copy of $F_{i-1}$ and the consecutive copy of $H_{i-1}$ induces a copy of $G_{i-1}$. Therefore we must have $\chi_{c}\left(T_{i+1}\right)=p_{i-1} / q_{i-1}$. Using the same argument as before (see the proof of the fact that $\left.\chi_{c}\left(G_{i}\right)=p_{i} / q_{i}\right)$, we conclude that for any $\left(p_{i-1}, q_{i-1}\right)$-colouring $\Delta$ of $T_{i+1}$, the restriction of $\Delta$ to the union of $F_{i}$ and the first copy of $F_{i-1}$ in $F_{i+1}$ is indeed a $\left(p_{i-1}, q_{i-1}\right)$-colouring of $T_{i}$, contrary to our assumption that $\chi_{c}\left(T_{i}\right)>p_{i-1} / q_{i-1}$. This completes the proof of Theorem 3.1.

\section{Counting the number of Edges}

In this section, we shall prove Theorems 1.1 and 1.2 , by counting the number edges of $M(p, q)$ for special values of $p$ and $q$.

PROOF OF THEOREM 1.1: It has been proved, at the beginning of Section 2, that if $q=1$ or $p=2 q+1$ then $G_{p}^{q}$ is edge critical. It remains to show that if $q \neq 1$ and $p \neq 2 q+1$ then $G_{p}^{q}$ is not edge critical. As $M(p, q)$ is a subgraph of $G_{p}^{q}$ with $\chi_{c}(M(p, q))=p / q$, it suffices to show that $M(p, q) \neq G_{p}^{q}$. This is obvious, because $G_{p}^{q}$ is regular, but when $q \neq 1$ and $p \neq 2 q+1, M(p, q)$ is not regular.

ProOF OF THEOREM 1.2: First we consider the case that $r$ is rational. Let $2 \leqslant$ $m<r \leqslant m+1$ be any rational number. Let $\left(\alpha_{1}, \alpha_{2}, \ldots, \alpha_{n}\right)$ be the alpha sequence of $r$. Note that when $r=m+1$, then we let the alpha sequence be (1). This does not satisfy the definition of alpha sequence, however, all the argument below are still valid.

For each $i \geqslant 1$, let $r_{i}$ be the rational number corresponds to the alpha sequence $\left(\alpha_{1}, \alpha_{2}, \ldots, \alpha_{n}+1,2, \ldots, 2\right)$, whose first $(n-1)$ entries coincide with the first $(n-1)$ entries of the alpha sequence of $r$, the $n$th entry is equal to 1 plus the $n$th entry of the alpha sequence of $r$, and the last $i$ entries are equal to 2 . In particular, when $r=m+1$, then the alpha sequence of $r_{i}$ has $i+1$ entries, all equal to 2 . 
Let

$$
m / 1=p_{0} / q_{0}<p_{1} / q_{1}<\cdots<p_{n-2} / q_{n-2}<p_{n-1} / q_{n-1}<p_{n} / q_{n}=r
$$

be the Farey sequence of $r$, and let

$$
m / 1=p_{0}^{\prime} / q_{0}^{\prime}<p_{1}^{\prime} / q_{1}^{\prime}<\cdots<p_{n}^{\prime} / q_{n}^{\prime}<p_{n+1}^{\prime} / q_{n+1}^{\prime}<\cdots<p_{n+i}^{\prime} / q_{n+i}^{\prime}=r_{i}
$$

be the Farey sequence of $r_{i}$. Then $p_{j}^{\prime}=p_{j}$ and $q_{j}^{\prime}=q_{j}$ for $j=0,1, \ldots, n-1$. Moreover, by applying $(* *)$, it is straightforward to verify that

$$
p_{n}=\alpha_{n} p_{n-1}-p_{n-2}, \quad p_{n}^{\prime}=\left(\alpha_{n}+1\right) p_{n-1}-p_{n-2}
$$

and that for $j=1,2, \ldots, i$, we have

$$
p_{n+j}^{\prime}=(j+1)\left(\left(\alpha_{n}+1\right) p_{n-1}-p_{n-2}\right)-j p_{n-1}=(j+1) p_{n}+p_{n-1}
$$

and similarly

$$
q_{n+j}^{\prime}=(j+1) q_{n}+q_{n-1} .
$$

Therefore

$$
r_{i}=\frac{(i+1) p_{n}+p_{n-1}}{(i+1) q_{n}+q_{n-1}}<p_{n} / q_{n}
$$

and hence $\lim _{i \rightarrow \infty} r_{i}=r$.

As we counted before, the number of vertices of $M\left(p_{n+i}^{\prime}, q_{n+i}^{\prime}\right)$ is equal to the number of vertices of $G_{p_{n+i}^{\prime}}^{q_{n+i}^{\prime}}$, which is $p_{n+i}^{\prime}$. Now we shall count the number of edges of the graphs $M\left(p_{n+i}^{\prime}, q_{n+i}^{\prime}\right)$ and $G_{p_{n+i}^{\prime}}^{q_{n+i}^{\prime}}$.

In the graph $G_{p_{n+i}^{\prime}}^{q_{n+i}^{\prime}}$, each vertex has degree $p_{n+i}^{\prime}-2 q_{n+i}^{\prime}+1=(i+1)\left(p_{n}-2 q_{n}\right)+$ $p_{n-1}-2 q_{n-1}+1$. Hence the number of edges is equal to

$$
\frac{1}{2}\left((i+1) p_{n}+p_{n-1}\right)\left((i+1)\left(p_{n}-2 q_{n}\right)+p_{n-1}-2 q_{n-1}+1\right)
$$

which has order $O\left((i+1)^{2}\right)$ as $i$ goes to infinity.

Let $F_{j}$ and $H_{j}$ be the graphs constructed as described in Section 2, by using the alpha sequence of $r_{i}$. Let $a, b$ and $c$ be the numbers of edges of the graphs $F_{n}, H_{n}$ and $F_{n+1}$ respectively. Then for $j=1,2, \ldots, i, F_{n+j}$ has $a$ edges if $j$ is even, and has $c$ edges if $j$ is odd. Let $e_{j}$ be the number of edges of $H_{n+j}$. Then $e_{j}=e_{j-1}+2(a+2 m-2)$ when $j$ is odd, and $e_{j}=e_{j-1}+2(c+2 m-2)$ when $j$ is even. The number of edges of $M\left(p_{n+i}^{\prime}, q_{n+i}^{\prime}\right)$ is equal to $e_{i}+a+2 m-2$ when $i$ is even, and is equal to $e_{i}+c+2 m-2$ when $i$ is odd. An explicit formula for this number can be found by solving the difference equation above. However, we shall not bother to solve it, just to observe that $e_{i}-e_{i-1}$ is 
bounded by a constant. Therefore the number of edges of $M\left(p_{n+i}^{\prime}, q_{n+i}^{\prime}\right)$ has order $O(i)$ when $i$ goes to infinity. Hence

$$
\left|E\left(M\left(p_{n+i}^{\prime}, q_{n+i}^{\prime}\right)\right)\right|=O\left(\sqrt{\left|E\left(G_{p_{n+i}^{\prime}}^{q_{n+i}^{\prime}}\right)\right|}\right) .
$$

This completes the proof of Theorem 1.2 for the case that $r$ is rational. If $r$ is irrational, then we let $s_{i}$ be rationals less than $r$ but approaching $r$. For each of the rationals $s_{i}$, we construct the corresponding sequence of graphs as above, then we use the diagonal method to choose one graph from each of these sequences of graphs. It is obvious that the resulting sequence of graphs gives a proof of Theorem 1.2 in this case.

When $m \geqslant 3$, we know that some more edges can be deleted from $M(p, q)$ without decreasing the circular chromatic number. However, we do not know if the order could be reduced to be smaller than $O\left(\sqrt{\left|E\left(G_{p}^{q}\right)\right|}\right)$. On the other hand, let $H$ be a subgraph of $G_{p}^{q}$ with the least number of edges such that $\chi_{c}(H)=p / q$. We do not know any non-trivial lower bound for the number of edges of $H$.

\section{REFERENCES}

[1] H.L. Abbott and B. Zhou, 'The star chromatic number of a graph', J. Graph Theory 17 (1993), 349-360.

[2] J.A. Bondy and P. Hell, 'A note on the star chromatic number', J. Graph Theory 14 (1990), 479-482.

[3] D. Duffus and N. Sauer, 'Lattice arising in categorical investigations of Hedetniemi's conjecture', Discrete Math. 152 (1996), 125-139.

[4] D.R. Guichard, 'Acyclic graph coloring and the complexity of the star chromatic number', J. Graph Theory 17 (1993), 129-134.

[5] D. Moser, 'The star-chromatic number of planar graphs', J. Graph Theory 24 (1997), 33-43.

[6] J. Nešetřil and X. Zhu, 'Path homomorphisms', Math. Proc. Cambridge Philos. Soc. 120 (1996), 207-220.

[7] E. Steffen and X. Zhu, 'On the star chromatic numbers of graphs', Combinatorica 16 (1996), 439-448.

[8] C. Tardif, 'Fractional multiples of graphs and the density of vertex-transitive graphs', (preprint).

[9] A. Vince, 'Star chromatic number', J. Graph Theory 12 (1988), 551-559.

[10] E. Welzl, 'Color-families are dense', J. Theoret. Comput. Sci. 17 (1982), 29-41.

[11] E. Welzl, 'Symmetric graphs and interpretations', J. Combin. Theory Ser. B 37 (1984), 235-244.

[12] X. Zhu, 'Star chromatic numbers and products of graphs', J. Graph Theory 16 (1992), 557-569.

[13] X. Zhu, 'Uniquely $H$-colorable graphs with large girth', J. Graph Theory 23 (1996), 33-41. 
[14] X. Zhu, 'Construction of uniquely $H$-colorable graphs', J. Graph Theory (to appear).

[15] X. Zhu, 'Planar graphs with circular chromatic numbers between 3 and 4', (preprint).

[16] X. Zhu, 'Circular chromatic number, a survey', (preprint).

Department of Applied Mathematics

National Sun Yat-sen University

Kaohsiung, Taiwan 80424

e-mail: zhu@math.nsysu.edu.tw 